OPEN ACCESS

Edited by:

Tomoharu Sugie,

Kansai Medical University Hospital,

Japan

Reviewed by:

Shinichiro Kashiwagi,

Osaka City University, Japan

Bin Yuan,

Anhui Medical University, China

${ }^{*}$ Correspondence:

Min Ma

mamin020@163.com

${ }^{\dagger}$ These authors have contributed equally to this work

Specialty section: This article was submitted to Breast Cancer,

a section of the journa

Frontiers in Oncology

Received: 18 October 2021 Accepted: 30 November 2021

Published: 17 January 2022

Citation:

Qiu D, Zhang G, Yan X, Xiao X, Ma $X$, Lin S, Wu J, Li X, Wang $W$,

Liu J, Ma Y and Ma M (2022) Prospects of Immunotherapy for Triple-Negative Breast Cancer.

Front. Oncol. 11:797092. doi: 10.3389/fonc.2021.797092

\section{Prospects of Immunotherapy for Triple-Negative Breast Cancer}

\author{
Dan Qiu ${ }^{1 \dagger}$, Guijuan Zhang ${ }^{2 \dagger}$, Xianxin Yan ${ }^{1 \dagger}$, Xinqin Xiao ${ }^{1}$, Xinyi Ma ${ }^{3}$, Shujun Lin $^{1}$, \\ Jieyan Wu ${ }^{1}$, Xinyuan $\mathrm{Li}^{4}$, Wandi Wang ${ }^{4}$, Junchen $\mathrm{Liu}^{4}$, Yi Ma ${ }^{5}$ and Min Ma ${ }^{1,6^{*}}$ \\ 1 School of Traditional Chinese Medicine of Jinan University, Jinan University, Guangzhou, China, 2 School of Nursing of Jinan \\ University, Jinan University, Guangzhou, China, ${ }^{3}$ School Public Health, Southern Medical University (No: 3210090112), \\ Guangzhou, China, ${ }^{4}$ School of Medicine, Jinan University, Guangzhou, China, ${ }^{5}$ Department of Cellular Biology, Institute of \\ Biomedicine, National Engineering, Research Center of Genetic Medicine, Key Laboratory of Bioengineering Medicine of \\ Guangdong Province, The National Demonstration Center for Experimental Education of Life Science and Technology, Jinan \\ University, Guangzhou, China, ${ }^{6}$ The First Affiliated Hospital of Jinan University, Jinan University, Guangzhou, China
}

In the classification and typing of breast cancer, triple-negative breast cancer (TNBC) is one type of refractory breast cancer, while chemotherapy stays in the traditional treatment methods. However, the impact of chemotherapy is short-lived and may lead to recurrence due to incomplete killing of tumor cells. The occurrence, development, and relapse of breast cancer are relevant to $T$ cell dysfunction, multiplied expression of related immune checkpoint molecules (ICls) such as programmed death receptor 1 (PD-1), programmed cell death 1 ligand 1 (PD-L1), and cytotoxic T-lymphocyte-associated antigen 4 (CTLA-4) produce immunosuppressive effect. Immunotherapy (namely, immune checkpoint inhibitors, adoptive cellular immunotherapy, CAR-T immunotherapy and some potential treatments) provides new hope in TNBC. This review focuses on the new immune strategies of TNBC patients.

Keywords: triple-negative breast cancers, immunotherapy, immune checkpoint molecules, PD1/PD-L1 pathway, CTLA-4, combination therapy

\section{INTRODUCTION: TREATMENT AND PROGNOSIS OF TNBCs}

According to the statistics of the World Health Organization (WHO), approximately 8.2 million people beings die of most cancers every year, accounting for $13 \%$ of international deaths. As one of the oldest tumors in the records of human civilization, breast cancer is the most clinically diagnosed cancer (1). In the classification and classification of breast cancer, breast cancer that does not express estrogen receptor (ER) or the progesterone receptor (PR) and does not amplify ERBB2 [commonly called human epidermal growth factor receptor 2 (HER2)] amplification are categorized as triplenegative breast cancer (TNBC), accounting for $10-20 \%$ of all breast cancers. TNBC subtypes were categorized by multi-omics data (2): (1) Intracavity androgen receptor subtype characterized by means of androgen receptor signal (23\%); (2) Immunomodulatory (IM) subtype (accounting for $24 \%$ of tumors) with excessive immune cell signal and cytokine signal gene expression; (3) A basallike and immune-suppressed (BLIS) (39\%) subtype, characterized with the aid of upregulation of cell cycle, activation of DNA restore and downregulation of immune response genes; and (4) a mesenchymal-like (MES) subtype rich in breast stem cell pathway (15\%). In addition, in the clinical patient population, we can see that TNBC is more common in young female patients. The tumor is usually large in size and of high grade, with greater lymph node metastasis at diagnosis, and has a 
high biological aggressiveness. Compared with women with other breast cancer subtypes, female with TNBC have higher early distant recurrence rate and worse 5-year prognosis. Therefore, it is very indispensable to obtain the cure purpose at an early stage or manipulate disorder inside the controllable range. Currently, the essential scientific remedies for TNBC consist of surgical resection, chemotherapy, radiotherapy, targeted therapy (3). Conventional chemotherapy drugs, including paclitaxel, anthracycline and alkylating agents, are prone to systemic toxicity and side effects. In addition, as patients with advanced TNBC are highly metastatic and aggressive, it is difficult to achieve good results with targeted therapy or hormone therapy alone (4).

\section{TNBCs AND IMMUNOTHERAPY}

In general, the immune system of healthy individuals is strong enough to shortly get rid of the mutated most cancers cells, while the immune function of most cancer patients can't successfully recognize and kill tumor cells (5). On the other hand, most tumor cells have many distinct mechanisms to defend them from being identified by means of immune cells (6). Different from the traditional therapies mentioned above, immunotherapy cannot efficaciously kill most tumor cells alone, however additionally decorate the immunity of patients, in particular in the removal of minimal residual lesions and drug-resistant tumor cells. It can keep away from many shortcomings of other therapies to the greatest extent (7). Cell immunotherapy, as a new technology with targeted killing effect on tumor cells, has achieved good results in clinical application in recent years.

\section{Immune Checkpoint Inhibitors}

ICIs are inhibitory molecules expressed on the cell surface, which are usually involved in regulating the activation of $\mathrm{T}$ cells. Basically, its most essential feature is comparable to the braking device of an auto-cell, which makes it "brake" in time when the immune system is activated, continues the activation of the immune system within normal limits, and avoids overactivation of the immune system. No matter whether overexpression or over-function of immune checkpoint molecules leads to suppression of immune function, resulting in low immunity and susceptibility to tumor and other diseases (8). Another way to think about it is that if the immunosuppressive function of checkpoint molecules is poor, the immune function of the body will be abnormal. Recent studies have shown that molecular pathways of immune checkpoints, such as programmed death ligand 1 (PD-L1) and programmed death ligand 2 (PD-L2), play a very important negative regulatory role in tumor immunity (9-11).

\section{CTLA-4 and TNBC}

It is conventional that CTLA-4 is a negative regulator, which is very vital for T cell-mediated immunity. In T cells, CTLA-4 and CD28 bind to the equal ligands (CD80 and CD86) on antigen imparting cells and have contrary effects. The interplay between
CTLA-4 and its ligand inhibits T cell reaction, and when CD28 and its ligand bind, $\mathrm{T}$ cell reaction is activated. The affinity of CTLA-4 to CD80/CD86 is greater than that of CD28 (12). The upregulation of CTLA-4 in cancer patients is considered as an important mediator of immune escape. Studies have shown that tumor cells of TNBC patients express CTLA-4 in different cell compartments (13). Its foremost ligand, CD80/CD86, is expressed in TNBC cell lines and tumors. This means that blocking CTLA-4 with Ipilimumab (anti-CTLA-4 monoclonal antibody, which has been accredited as checkpoint inhibitor for melanoma treatment) can significantly activate the molecular cascade, which may help enhance the immune response to tumor cells (14). CTLA-4 expressed on the surface of tumor cells during the treatment of patients with TNBC may be the target of checkpoint inhibitors and a candidate biomarker for immunotherapy. In a word, we believe that the operation or chemotherapy of TNBC patients, not only can the combination of targeted immune checkpoint drug therapy play a synergistic role to a great extent, but also can increase the cure probability of cancer patients.

\section{PD-1 and PD-L1}

PD-1 antibody is a most researched and clinically developed immunotherapy. PD-1 is expressed in activated T cells, B cells, and myeloid cells. It has two ligands, PD-L1 and PD-L2. The binding of PD-1 and PD-L1 mediates the co-inhibitory signal of $\mathrm{T}$ cell activation, suppresses the killing function of $\mathrm{T}$ cells, and performs a negative regulatory role in human immune response (15-17). In a normal immune system, PD-1 is up to preserve the position of immune tolerance. Tumor cells can escape immune surveillance through immune escape. Targeted therapy based on immunosuppressive receptors and immunosuppressive checkpoint immunotherapy based on immune molecules are new hotspots in oncology research $(18,19)$. It is additionally discovered that PD-L1 binds to PD-1 receptor on activated T cells and weakens anti-tumor immunity by inhibiting $\mathrm{T}$ cell activation signal. PD- $1^{+}$T cells can partially recover by blocking PD-1/PD-L1 signaling pathway (20-24). Some studies have proven that PD-L1 antibody combined with paclitaxel is effective in treating advanced in the treatment of advanced TNBCs (25). TNBC subtype research based on multi-group data shows that immunoglobulin subtype has high immune cell signal (2). Both clinical and economic characteristics indicate that immune recognition is activated in IM subtype, which shows that the mechanism of immune break out of these tumors may additionally contain the recruitment of immunosuppressive cells or the activation of immune checkpoint molecules. Based on what has been discussed above, we may conclude that high expression levels of immune checkpoint suppressor genes such as PD1, PD-L1, cytotoxic Tlymphocyte-associated antigen 4 (CTLA4), and IDO1 (Indoleamine 2,3-dioxygenase 1) may inhibit the activation of the immune system and lead to the occurrence of TNBC. A Phase III trials confirmed that Atezizumab (PD-L1 inhibitor) and nabo-paclitaxel in the treatment of advanced TNBC, compared with placebo + nabo-paclitaxel, atezizumab + 
nabo- Paclitaxel can significantly improve the progression-free survival (PFS) (7.5 months vs 5.5 months, respectively) and overall survival (OS) (25.0 months vs 15.5 months, respectively) (26). The response rate of TNBC to ICIs is higher than that of hormone-receptor positive and HER2positive breast cancers. Recently, the inhibitory effect of carbamazepine plus apatinib on PD-1 in advanced patients in Phase II trial was found, and carbamazepine plus apatinib had good tolerance and showed good ORR (43.3\%) and PFS in advanced patients, regardless of lines of therapy and PD-L1 status [NCT03394287] (27). Furthermore, PD-1/PD-L1 inhibitors combined with chemotherapy are more successful in TNBC than single dose ICIs. These results indicate that combinations with chemotherapy could increase the response rate to immunotherapy compared to chemotherapy or immune checkpoint blockade alone.

We summarize the relevant clinical studies in the treatment of TNBC by ICIs in recent years (Figures 1-3) (28-43).

\section{Adoptive Cellular Immunotherapy (CD8 ${ }^{+}$T Cells)}

The tumor immune microenvironment (TIME) plays a critical role in the progression, response to therapy and prognosis of most cancer patients. Tumor-infiltrating lymphocytes (TIL) are one of the predominant components of TIME, and the density and types of lymphocytes in the TIL fraction of a tumor have marked prognostic associations in breast cancer. This is especially actual of TNBC, which has the largest number of TILs. CD8 T cells are necessary immune cell in TIL (44). The infiltration of $\mathrm{CD}^{+} \mathrm{T}$ lymphocytes into solid tumors is related to the good prognosis of various types of cancers including TNBC (45). The $\mathrm{T}$ cell antigen receptor (TCR) of $\mathrm{CD}^{+} \mathrm{T}$ cells recognizes an antigenic peptide containing approximately 1317 amino acids. It consists of major histocompatibility complex I molecules (MHC-I). Some research have observed that in TNBC transgenic mouse model, the levels of IFN- $\gamma$ and TNF- $\alpha$ increased tumor-invasive $\mathrm{CD}^{+} \mathrm{T}$ cells, and subsequently led to apoptosis (46). Inhibition of Tregs (Regulatory cells) amplification can enhance the anti-tumor response of $\mathrm{CD}^{+} \mathrm{T}$ cells, thus affecting the growth of primary breast tumors or the metastasis of cancer cells to the lung $(47,48)$. Some studies have shown that LXR-inverse (Liver-X-Receptors) activation stimulates immune-mediated tumor destruction by means of improving CD8 T-cell activity in TNBC (49). It has currently been proved that PARP inhibitor olaparib induces $\mathrm{CD}^{+} \mathrm{T}$ cell infiltration in TNBC model with a BRCA1-deficient (breast cancer 1) deficiency by activating STING (interferon gene) pathway. Similarly, the efficacy of PARP inhibitors depends on the recruitment of $\mathrm{CD}^{+} \mathrm{T}$ cells in BRCA deficient TNBC model by activating intracavitary STING pathway $(50,51)$. STINGtargeted immunotherapy enhances anti-tumor immunity mediated by natural killer cells and $\mathrm{CD} 8^{+} \mathrm{T}$ cells. It provides a theoretical basis for combining PARP inhibitors with CAR-T (Chimeric antigen receptor $\mathrm{T}$ ) cell remedy to deal with TNBC disease.

\section{CAR-T and CAR-NK}

CAR-T immunotherapy, which directly retargets the immune system od the patient to perceive and eradicate tumor cells with tumor-associated antigens (TAAs), and is presently being explored as a treatment for TNBCs $(52,53)$. However, immunotherapy is a new technology, and many bottlenecks remain to be overcome. For example, identifying specific goal tumor antigens and designing effective CAR is one of the many challenges of CAR-T therapy. Studies have shown that epidermal growth factor receptor-CAR (EGFR-CAR) lentivirus-infected $\mathrm{T}$ cells have a robust specific inhibitory effect on the growth of TNBC cells and tumor occurrence in vitro and in vivo (54). Some $s$ find out about exhibit that ICAM1 (intercellular adhesion molecule-1)-specific CAR-T cells have been in a position to efficiently recognize ICAM1 expressing TNBC cells, and they can effectively minimize the growth of TNBC tumor inside and outside (55). Recently, the University of Pennsylvania completed a first phase scientific trial, which studied the security of injecting c-Met-CAR-T cell into TNBC patients [NCT01837602] (56). Results Inflammatory reaction was induced in TNBC tumor, and there was no evidence that drugrelated side effects were greater than grade 1 . Up to now, the research on MUC1 (Mucin1 glycoprotein)-CAR-T cell therapy has been the most investigated in clinical trials (57). The safety and efficacy of autologous MUC1-CAR-T cells are proposed to be evaluated in a phase I/II study in patients with relapsed or refractory TNBC [NCT02587689] (56). In addition, the inhibition of TGF- $\beta$-receptor signaling augments the antitumor function of ROR1 (receptor-tyrosine-kinase-like orphan receptor 1)-specific CAR T-cells against TNBC (58). Moreover,

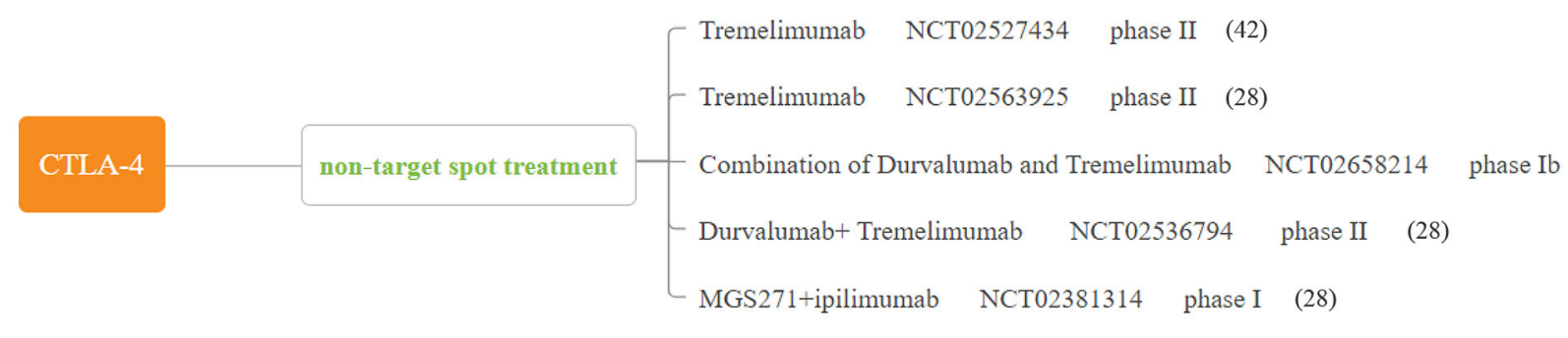

FIGURE 1 | Major published clinical trials using CTLA-4 inhibitors in TNBC. 


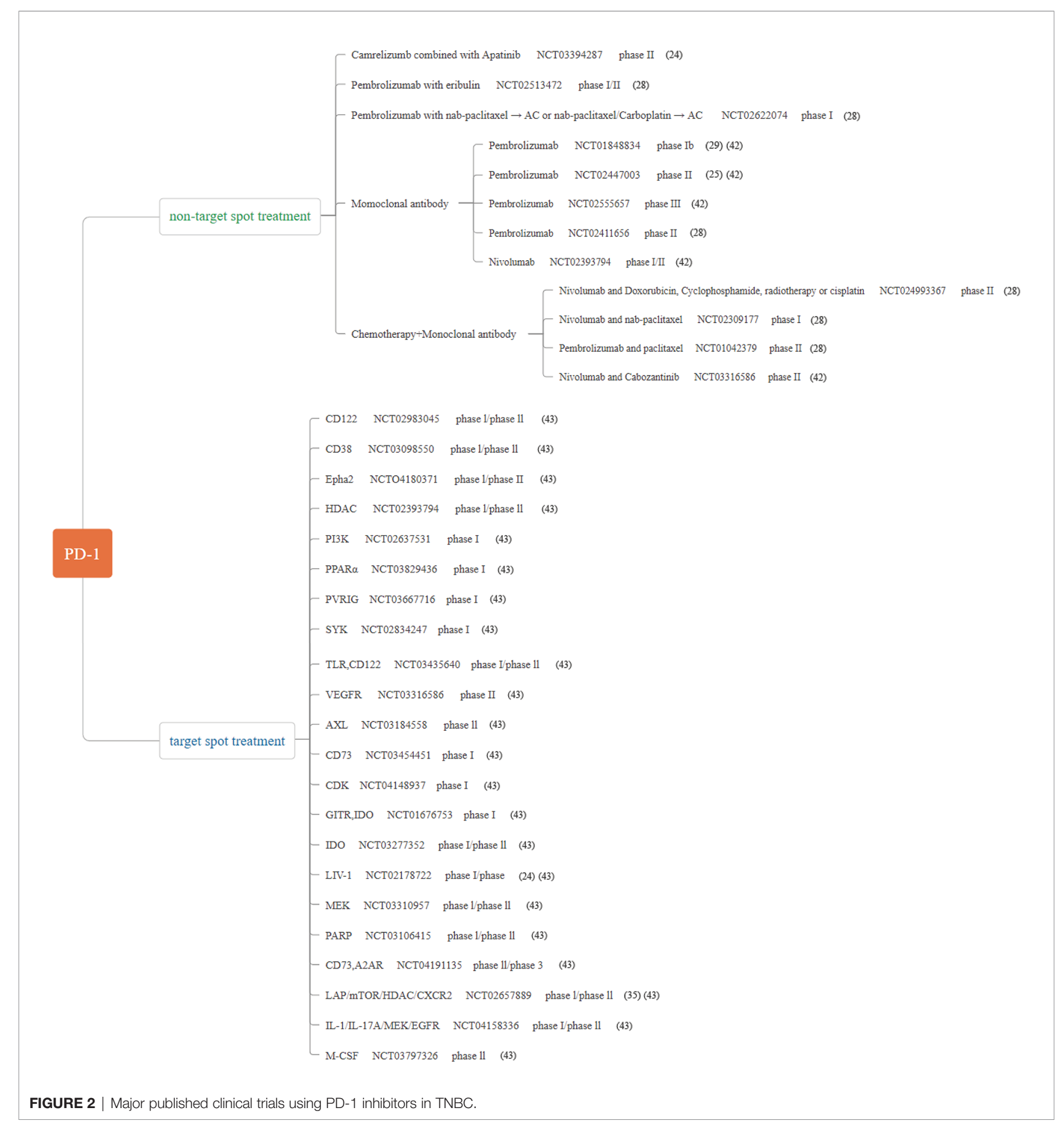

when recruiting participants, the safety and tolerance of allogeneic gamma delta $(\gamma \delta) \mathrm{T}$ cells transduced with CARs targeting NKG2D ligands on TNBC cells will be investigated in Phase I clinical trials [NCT04107142] $(59,60)$. The number of clinical trials of TNBC that CAR-T cell therapy is increasing, which may produce some exciting clinical effects. In addition, NK cells play a prominent role in the innate immune system because multiple receptors on the surface of the NK cells have been approved to kill cancer cells by interacting with their ligands of cancer cells, leading to apoptosis o cancer cells. Studies have shown that tissue factor as a new target for CARNK cell immunotherapy of TNBC (61). As EGFR is a potential therapeutic target for TNBC, EGFR-specific CAR NK cells (EGFR-CAR NK cells) is a promising strategy to inhibit tumor growth in breast cancer cell line-derived xenograft (CLDX) and patient-derived xenograft (PDX) mouse models (62). 


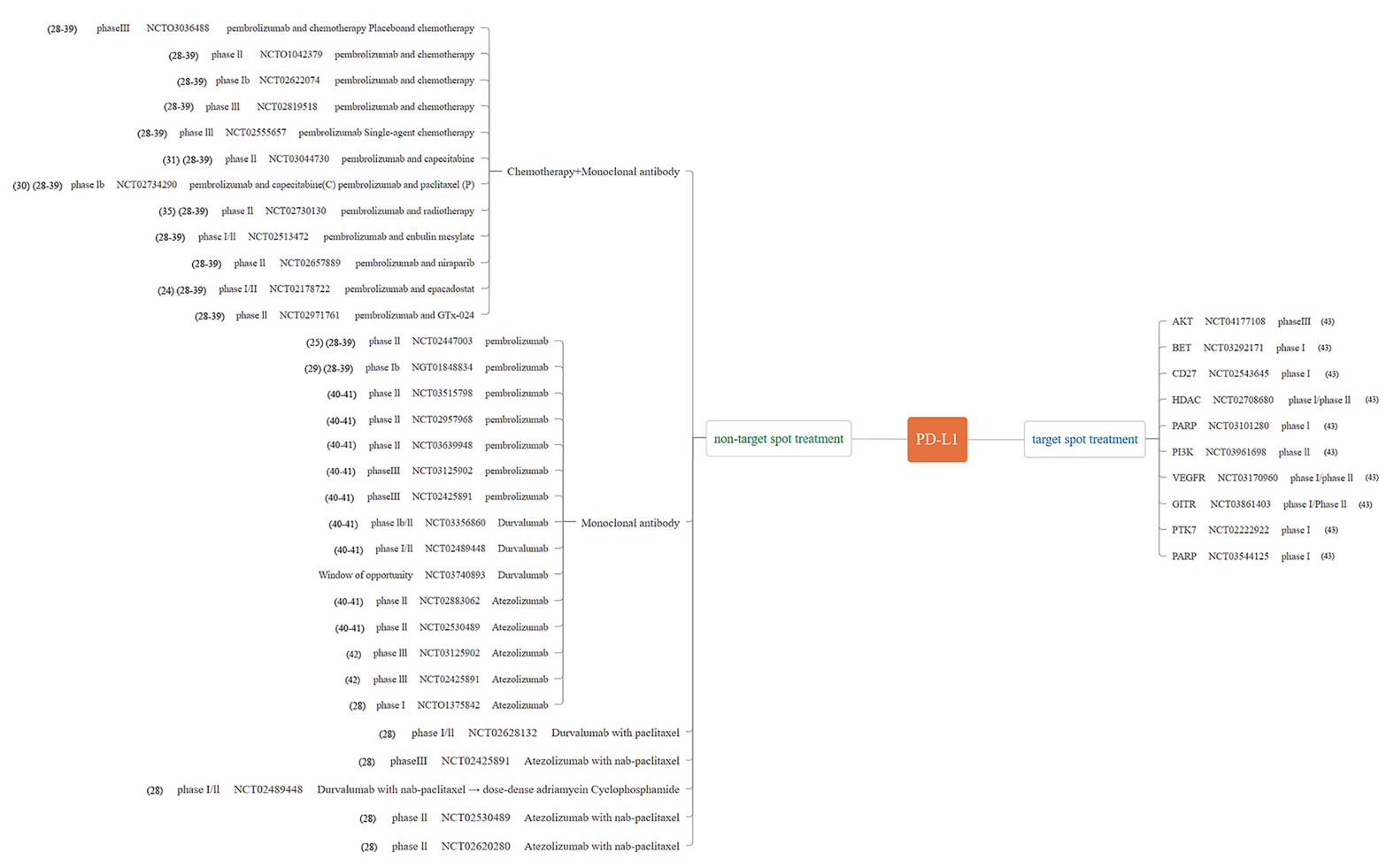

FIGURE 3 | Major published clinical trials using PD-L1 inhibitors in TNBC.

\section{COMBINATION THERAPY}

In targeted therapy of TNBC, there are some small molecule therapeutic targets, namely, PARP, DNA (cytosine-5)methyltransferase 1(DNMT1), epidermal growth factor (EGF) and EGF receptor (EGFR), fibroblast growth factor receptor (FGFR), vascular endothelial growth factor (VEGF), and VEGF receptor (VEGFR) $(63,64)$. In TNBC subtype, basic helix-loophelix (bHLH) transcription factors inhibitor of differentiation 1 (ID1) and inhibitor of differentiation 3 (ID3) (referred to as Id) play a vital role in maintaining cancer stem cell (CSC). Many molecules have been in preclinical trials. The application of ispinesib (a small molecule inhibitor in the ID1+ CSC results) to target the ID/Kif11 pathway, combined with chemotherapy, gave better response in TNBC subtype (65). This targeting ID1Kif11 molecular pathway in the ID1+ CSCs, combined with chemotherapy and small molecular inhibitor, may reduce TNBC effect more effectively.

In addition, another promising strategy for combination therapy is to turn the "cold" tumors "hot" (66). Through a variety of methods, such as attracting $\mathrm{T}$ cell to the tumor through chemotherapy, radiation therapy, vaccines, and oncolytic viruses and bispecific antibodies (67). Other combination strategies include inhibition of other checkpoints or other immunosuppressive mechanisms, or enhancement of the activity of other checkpoint agonists, combination therapy to overcome $\mathrm{T}$ cell exhaustion, or conversion of immunosuppression [e.g., regulatory $\mathrm{T}$ cells (Tregs), myeloid-derived suppressor cells] into immunoreactive phenotypes $(68,69)$. In TNBC, however, chemotherapy combination of Atezolizumab enhanced the antitumor efficacy of Nab-paclitaxel only in patients with PD-L1 expression on tumor-infiltrating immune cells [NCT03371017] (26). On the other hand, chemotherapy combination of pembrolizumab paclitaxel protein-bound, or paclitaxel, or gemcitabine plus carboplatin also benefit patients with TNBC [NCT02819518] (70). Above all, tumors that respond to immune checkpoint inhibitors are typically so-called thermal or "hot" tumors with CD8 $\mathrm{T}$ cell infiltration, indicating that tumor cells are recognized by the immune system. CD8 positivity is often assessed as a predictor of response and a pharmacodynamic marker of response to combination therapies, which are hypothesized to enhance T cell infiltration and heat so-called "cold" tumors (71). Similarly, TNBC features immunological "cold" tumor, which with limited tumor infiltrating lymphocytes (72). To address this problem, we need to find a methodological strategy that actively recruits $\mathrm{CD}^{+} \mathrm{T}$ cells into the tumor microenvironment (TME), reverses "cold" tumors into "hot" tumors, and significantly improves their reactivity to ICIs (73).

\section{POTENTIAL THERAPEUTIC DIRECTIONS AND POSSIBLE STRATEGIES}

\section{Mesothelin and TNBC}

Tumor-associated antigen-mesothelin (MSLN) is a glycoprotein that exists on the cell surface and is highly expressed in various 


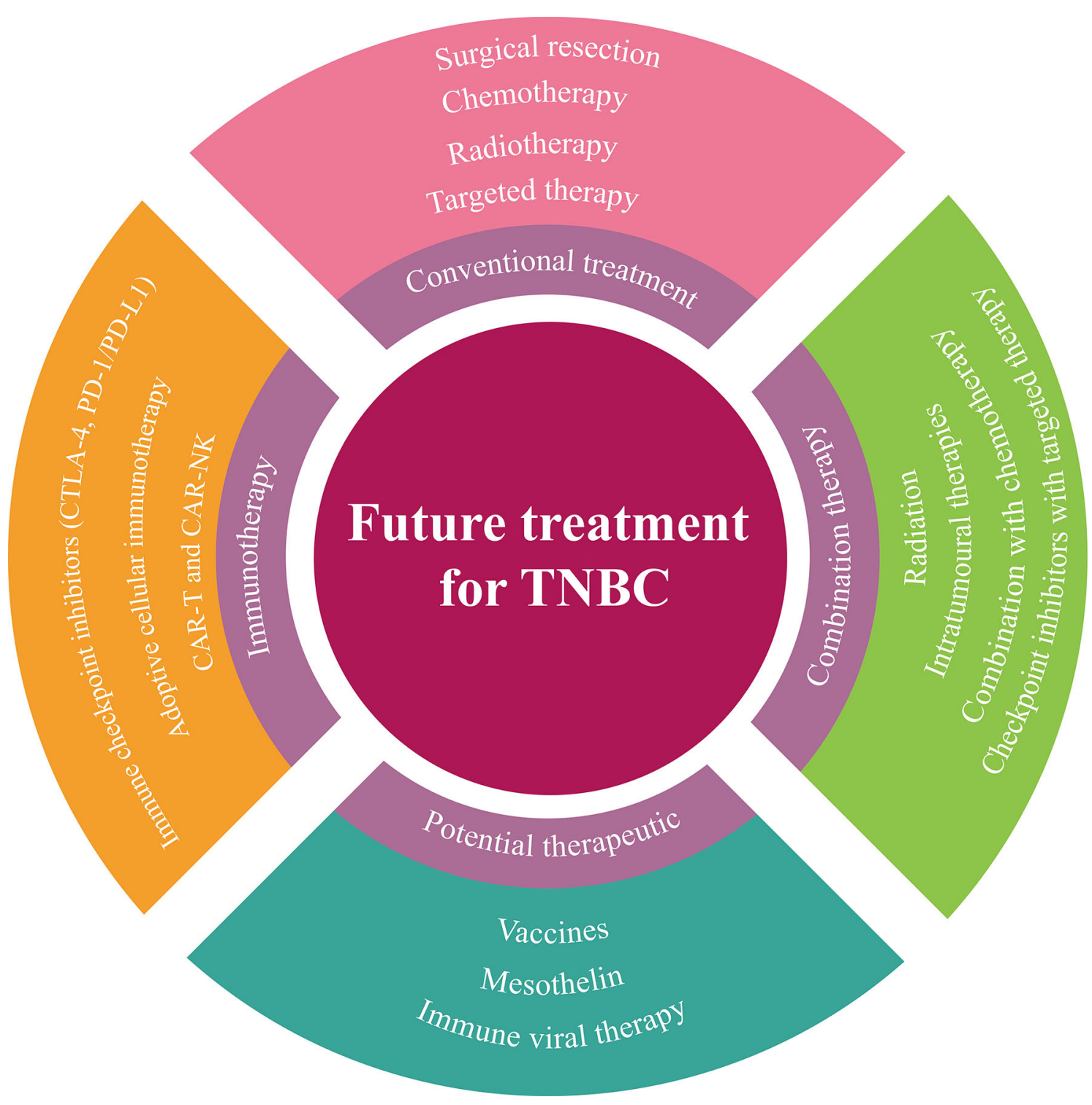

FIGURE 4 | A summary of future treatment strategies for TNBC.

tumor tissues such as mesothelioma, non-small cell lung cancer, pancreatic cancer, and metastatic triple negative breast cancer, while no longer expressed in normal tissues or is low expressed in mesothelial tissues (74). Due to the characteristic of MSLN, it has become the focus of specific targeting antigen of tumor cells. Recent lookup used to be discovery of MSLN, a carcinogenic glycosyl-phosphatidyl-inositol (GPI) is overexpressed in TNBC (75). Above all, MSLN additionally play an vital position in T cell cloning and expansion and effector function, including initiating T cell activation (76). MSLN immune-targeted therapy (mAbs, CAR-T, vaccine) has top notch potential, and many of them have entered clinical trials of pancreatic cancer and lung cancer (76, 77). As a new personalized therapy, MSLN targeted therapy may achieve positive clinical results in TNBC patients.

\section{TNBC and Immune Viral Therapy}

Recent trends in viral genetic engineering have allowed the development of oncolytic viruses with enhanced recognition capability to receptors overexpressed in tumor tissues, and viruses encoding or packaging suicide or pro-apoptotic genes or agents for delivery to cancer cells (78). Viruses can be manipulated to upregulate antigen presentation and $\mathrm{T}$ cell anti-tumor response. Talimogene laherparepvec (T-Vec, OncoVEXGM-CSF, Imlygic), an attenuated and genetically engineered herpes simplex virus (HSV) that overexpresses granulocyte-macrophage colony-stimulating factor (GM-CSF), is the only oncolytic virus approved for clinical use in the United States and Europe (ClinicalTrials.gov:NCT00769704) (79-81). Some studies have shown that cell vaccines primarily based on 
oncolytic vesicular stomatitis virus can improve the prognosis of TNBC by enhancing the functions of natural killer cells and $\mathrm{CD}^{+} \mathrm{T}$ cells (82). An oncolytic herpes simplex virus, which encodes the fundamental anti-tumor cytokine, interleukin 12 (IL-12), (designated G471-mIL12), can selectively kill cancer cells while inducing anti-tumor immunity (83), which is mainly manifested by the upregulation of $\mathrm{CD}^{+} \mathrm{T}$ cells activation markers in tumor microenvironment and the inhibition of tumor angiogenesis (84). Immunovirotherapy may be a promising method to treat TNBC patients.

\section{TNBC and Vaccines}

Some studies have shown that mixed 19-peptide vaccine alone can achieve positive results in the treatment of refractory TNBC (85). The multi-epitope DNA and peptide vaccines is composed of the most immune dominant epitopes of SYCP1 (Synaptonemal Complex Protein 1) and ACRBP (Acrosin Binding Protein). As two conventional cancer/testis antigens, it can effectively activate the cellular and humoral immune response against $4 \mathrm{~T} 1$ mouse breast tumor. In addition, this preventive combined immunization can drastically inhibit the growth of this mouse triple negative breast tumor (86). However, there are still some problems to be solved about vaccines, such as time, administration frequency and combination therapy strategy.

\section{CONCLUSIONS}

Recently, immunotherapy has delivered new hope to TNBC. The application of ICIs in TNBC will bring new light and advantage to patients. TNBC is currently exploring other new immunotherapy strategies, consisting of oncolytic virus and adoptive cell therapy, such as TIL metastasis and carcinoembryonic antigen $\mathrm{T}$ cells. Breast cancer vaccine constitutes another new therapeutic strategy to enhance anti-cancer immunity. Although the new preliminary immunotherapy still needs extensive clinical verification, these immunotherapies will promote the understanding of anti-cancer

\section{REFERENCES}

1. Bray F, Ferlay J, Soerjomataram I, Siegel RL, Torre LA, Jemal A. Global Cancer Statistics 2018: GLOBOCAN Estimates of Incidence and Mortality Worldwide for 36 Cancers in 185 Countries. CA Cancer J Clin (2018) 68 (6):394-424. doi: 10.3322/caac.21492

2. Jiang YZ, Ma D, Suo C, Shi JX, Xue MZ, Hu X, et al. Genomic and Transcriptomic Landscape of Triple-Negative Breast Cancers: Subtypes and Treatment Strategies. Cancer Cell (2019) 35(3):428. doi: 10.1016/j.ccell.2019.02.001

3. Xin L, Li JY, Yu ZG, Liu YH. Chinese Breast Cancer Surgery: Clinical Practice and Research. Chin Med J (2020) 133(19):2269-70. doi: 10.1097/ Cm9.0000000000000992

4. Giovannelli P, Di Donato M, Galasso G, Di Zazzo E, Bilancio A, Migliaccio A. The Androgen Receptor in Breast Cancer. Front Endocrinol (Lausanne) (2018) 9:492. doi: 10.3389/fendo.2018.00492

5. Smits BMG, Sharma D, Samuelson DJ, Woditschka S, Mau B, Haag JD, et al. The Non-Protein Coding Breast Cancer Susceptibility Locus Mcs5a Acts in a Non-Mammary Cell-Autonomous Fashion Through the Immune System and Modulates T-Cell Homeostasis and Functions. Breast Cancer Res (2011) 13 (4):R81. doi: 10.1186/bcr2933 immunity of breast cancer and contribute to the development of effective strategies in the future. Further understanding of the mechanisms underlying immu-oncology are warranted to identify new immunotherapy-sensitive tumor types, combinations of different therapies will also become a promising strategy in the treatment of TNBC (Figure 4).

\section{AUTHOR CONTRIBUTIONS}

DQ: Writing - original draft, Writing-Review \& editing. GZ, $\mathrm{XY}$, and XX: Resources, Writing-Original draft. XM, SL, JW, $\mathrm{XL}, \mathrm{WW}, \mathrm{JL}$, and YM: Participate in article writing. MM: Conceptualization, Writing-Original draft, Writing-Review \& editing, Project administration, Funding acquisition. All authors contributed to the article and approved the submitted version.

\section{FUNDING}

This work was supported by the National Natural Science Foundation of China (nos. 82074430, 81803979, 81673979, 82073748 and 81741130); the Natural Science Foundation of Guangdong Province, China (nos. 2018A030313393 and 2016A030313114); Science and Technology Program of Guangzhou, China (nos. 201803010051, 201707010245, and 201704020117) and the Fourth Batch of TCM Clinical Outstanding Talent Program of China (no. 444258); The Guangdong Basic and Applied Basic Research Foundation (no. 2019A1515011866 and 2021A1515010993); Guangdong Science and Technology Innovation Strategy Special fund (International science and technology cooperation projects, no. 2021A0505030034) Jinan University's the National Collegiate Innovation and Startups Training Program (no. 202110559093). Thanks for the general Project of Natural Science Foundation of Guangdong Province, China in 2022.

6. D’Arrigo P, Tufano M, Rea A, Vigorito V, Novizio N, Russo S, et al Manipulation of the Immune System for Cancer Defeat: A Focus on the T Cell Inhibitory Checkpoint Molecules. Curr Med Chem (2018) 27(15):240248. doi: 10.2174/0929867325666181106114421

7. Zhu MMT, Burugu S, Gao D, Yu J, Kos Z, Leung S, et al. Evaluation of Glucocorticoid-Induced TNF Receptor (GITR) Expression in Breast Cancer and Across Multiple Tumor Types. Mod Pathol (2020) 33(9):1753-63. doi: 10.1038/ s41379-020-0550-Z

8. Katz H, Alsharedi M. Immunotherapy in Triple-Negative Breast Cancer. Med Oncol (2017) 35(1):13. doi: 10.1007/s12032-017-1071-6

9. Pina-Sanchez P, Valdez-Salazar HA, Ruiz-Tachiquin ME. Circulating microRNAs and Their Role in the Immune Response in Triple-Negative Breast Cancer. Oncol Lett (2020) 20(5):224. doi: 10.3892/ol.2020.12087

10. Keenan TE, Tolaney SM. Role of Immunotherapy in Triple-Negative Breast Cancer. J Natl Compr Cancer Netw (2020) 18(4):479-89. doi: 10.6004/ jnccn.2020.7554

11. Fabrizio FP, Trombetta D, Rossi A, Sparaneo A, Castellana S, Muscarella LA. Gene Code CD274/PD-L1: From Molecular Basis Toward Cancer Immunotherapy. Ther Adv Med Oncol (2018) 10:1-18. doi: 10.1177/ 1758835918815598 
12. Navarrete-Bernal MGC, Cervantes-Badillo MG, Martinez-Herrera JF, LaraTorres CO, Gerson-Cwilich R, Zentella-Dehesa A, et al. Biological Landscape of Triple Negative Breast Cancers Expressing CTLA-4. Front Oncol (2020) 10:1206. doi: 10.3389/fonc.2020.01206

13. Pack CD, Bommireddy R, Munoz LE, Patel JM, Bozeman EN, Dey P, et al. Tumor Membrane-Based Vaccine Immunotherapy in Combination With AntiCTLA-4 Antibody Confers Protection Against Immune Checkpoint Resistant Murine Triple-Negative Breast Cancer. Hum Vaccines Immunotherapeut (2020) 16(12):3184-93. doi: 10.1080/21645515.2020.1754691

14. Bernier C, Soliman A, Gravel M, Dankner M, Savage P, Petrecca K, et al. DZ2384 has a Superior Preclinical Profile to Taxanes for the Treatment of TripleNegative Breast Cancer and Is Synergistic With Anti-CTLA-4 Immunotherapy. Anti-Cancer Drugs (2018) 29(8):774-85. doi: 10.1097/ Cad. 0000000000000653

15. Boussiotis VA. Molecular and Biochemical Aspects of the PD-1 Checkpoint Pathway. N Engl J Med (2016) 375(18):1767-78. doi: 10.1056/ NEJMra1514296

16. Tokumaru Y, Joyce D, Takabe K. Current Status and Limitations of Immunotherapy for Breast Cancer. Surgery (2020) 167(3):628-30. doi: 10.1016/j.surg.2019.09.018

17. Alsaab HO, Sau S, Alzhrani R, Tatiparti K, Bhise K, Kashaw SK, et al. PD-1 and PD-L1 Checkpoint Signaling Inhibition for Cancer Immunotherapy: Mechanism, Combinations, and Clinical Outcome. Front Pharmacol (2017) 8:561. doi: 10.3389/fphar.2017.00561

18. Conway JR, Kofman E, Mo SS, Elmarakeby H, Van Allen E. Genomics of Response to Immune Checkpoint Therapies for Cancer: Implications for Precision Medicine. Genome Med (2018) 10(1):93. doi: 10.1186/s13073-0180605-7

19. Krieger T, Pearson I, Bell J, Doherty J, Robbins P. Targeted Literature Review on Use of Tumor Mutational Burden Status and Programmed Cell Death Ligand 1 Expression to Predict Outcomes of Checkpoint Inhibitor Treatment. Diagn Pathol (2020) 15(1):6. doi: 10.1186/s13000-020-0927-9

20. Ribas A, Wolchok JD. Cancer Immunotherapy Using Checkpoint Blockade. Science (2018) 359(6382):1350. doi: 10.1126/science.aar4060

21. Vonderheide RH. The Immune Revolution: A Case for Priming, Not Checkpoint. Cancer Cell (2018) 33(4):563-9. doi: 10.1016/j.ccell.2018.03.008

22. Sun C, Mezzadra R, Schumacher TN. Regulation and Function of the PD-L1 Checkpoint. Immunity (2018) 48(3):434-52. doi: 10.1016/j.immuni.2018.03.014

23. Sun T, Zhang W, Li Y, Jin Z, Du Y, Tian J, et al. Combination Immunotherapy With Cytotoxic T-Lymphocyte-Associated Antigen-4 and Programmed Death Protein-1 Inhibitors Prevents Postoperative Breast Tumor Recurrence and Metastasis. Mol Cancer Ther (2020) 19(3):802-11. doi: 10.1158/1535-7163.MCT-19-0495

24. Zou WP, Wolchok JD, Chen LP. PD-L1 (B7-H1) and PD-1 Pathway Blockade for Cancer Therapy: Mechanisms, Response Biomarkers, and Combinations. Sci Trans Med (2016) 8(328):328rv4. doi: 10.1126/scitranslmed.aad7118

25. Planes-Laine G, Rochigneux P, Bertucci F, Chretien AS, Viens P, Sabatier R, et al. PD-1/PD-L1 Targeting in Breast Cancer: The First Clinical Evidences Are Emerging-A Literature Review. Cancers (2019) 11(7):1033. doi: 10.3390/ cancers 11071033

26. Schmid P, Adams S, Rugo HS, Schneeweiss A, Barrios CH, Iwata H, et al. Atezolizumab and Nab-Paclitaxel in Advanced Triple-Negative Breast Cancer. N Engl J Med (2018) 379(22):2108-21. doi: 10.1056/NEJMoa1809615

27. Liu JQ, Liu Q, Li Y, Li Q, Su FX, Yao HR, et al. Efficacy and Safety of Camrelizumab Combined With Apatinib in Advanced Triple-Negative Breast Cancer: An Open-Label Phase II Trial. J Immunother Cancer (2020) 8(1): e000696. doi: 10.1136/jitc-2020-000696

28. Adams S, Loi S, Toppmeyer D, Cescon DW, De Laurentiis M, Nanda R, et al. Pembrolizumab Monotherapy for Previously Untreated, PD-L1-Positive, Metastatic Triple-Negative Breast Cancer: Cohort B of the Phase II KEYNOTE-086 Study. Ann Oncol (2019) 30(3):405-11. doi: 10.1093/ annonc/mdy518

29. Adams S, Schmid P, Rugo HS, Winer EP, Loirat D, Awada A, et al. Pembrolizumab Monotherapy for Previously Treated Metastatic TripleNegative Breast Cancer: Cohort A of the Phase II KEYNOTE-086 Study. Ann Oncol (2019) 30(3):397-404. doi: 10.1093/annonc/mdy517

30. Cortes J, Lipatov O, Im SA, Goncalves A, Lee KS, Schmid P, et al. KEYNOTE119: Phase III Study of Pembrolizumab (Pembro) Versus Single-Agent
Chemotherapy (Chemo) for Metastatic Triple Negative Breast Cancer (mTNBC). Ann Oncol (2019) 30:859. doi: 10.1093/annonc/mdz394.010

31. Ho AY, Barker CA, Arnold BB, Powell SN, Hu ZSI, Gucalp A, et al. A Phase 2 Clinical Trialassessing Theefficacy and Safety of Pembrolizumab and Radiotherapy in Patients With Metastatic Triple-Negative Breast Cancer. Cancer (2020) 126(4):850-60. doi: 10.1002/cncr.32599

32. Lee-Bitar JS, Frankel PH, Yost SE, Synold TW, Martinez N, Tang AL, et al. A Phase II Clinical Trial of Pembrolizumab and Selective Androgen Receptor Modulator GTx-024 in Patients With Advanced Androgen Receptor-Positive Triple-Negative Breast Cancer. J Clin Oncol (2019) 37(15):1069. doi: 10.1200/ JCO.2019.37.15_suppl.1069

33. Nanda R, Chow LQM, Dees EC, Berger R, Gupta S, Geva R, et al. Pembrolizumab in Patients With Advanced Triple-Negative Breast Cancer: Phase Ib KEYNOTE-012 Study. J Clin Oncol (2016) 34(21):2460. doi: 10.1200/ Jco.2015.64.8931

34. Page DB, Chun B, Pucilowska J, Kim I, Sanchez K, Redmond WL, et al. Pembrolizumab (Pembro) With Paclitaxel (Taxol) or Capecitabine (Cape) as Early Treatment of Metastatic Triple-Negative Breast Cancer (mTNBC) J Clin Oncol (2019) 37(15):1015. doi: 10.1200/JCO.2019.37.15_suppl.1015

35. Shah AN, Flaum L, Helenowski I, Santa-Maria CA, Jain S, Rademaker A, et al. Phase II Study of Pembrolizumab and Capecitabine for Triple Negative and Hormone Receptor-Positive, HER2-Negative Endocrine-Refractory Metastatic Breast Cancer. J Immunother Cancer (2020) 8(1):e000173. doi: 10.1136/jitc-2019-000173

36. Spira AI, Hamid O, Bauer TM, Borges VF, Wasser JS, Smith DC, et al. Efficacy/safety of Epacadostat Plus Pembrolizumab in Triple-Negative Breast Cancer and Ovarian Cancer: Phase I/II ECHO-202 Study. J Clin Oncol (2017) 35:1103. doi: 10.1200/JCO.2017.35.15_suppl.1103

37. Tolaney SM, Kalinsky K, Kaklamani V, Savulsky C, Olivo M, Aktan G, et al. Phase 1b/2 Study to Evaluate Eribulin Mesylate in Combination With Pembrolizumab in Patients With Metastatic Triple-Negative Breast Cancer. Asia-Pac J Clin Oncol (2018) 14:176. doi: 10.1158/1538-7445.SABCS17-PD6-13

38. Vinayak S, Tolaney SM, Schwartzberg L, Mita M, McCann G, Tan AR, et al. Open-Label Clinical Trial of Niraparib Combined With Pembrolizumab for Treatment of Advanced or Metastatic Triple-Negative Breast Cancer. JAMA Oncol (2019) 5(8):1132-40. doi: 10.1001/jamaoncol.2019.1029

39. McArthur HL, Diab A, Page DB, Yuan JD, Solomon SB, Sacchini V, et al. A Pilot Study of Preoperative Single-Dose Ipilimumab and/or Cryoablation in Women With Early-Stage Breast Cancer With Comprehensive Immune Profiling. Clin Cancer Res (2016) 22(23):5729-37. doi: 10.1158/10780432.Ccr-16-0190

40. Cortes J, Cescon DW, Rugo HS, Nowecki Z, Im SA, Yusof MM, et al. Randomized, Double-Blind, Phase III Study of Pembrolizumab Plus Chemotherapy Versus Placebo Plus Chemotherapy for Previously Untreated Locally Recurrent Inoperable or Metastatic Triple-Negative Breast Cancer. J Clin Oncol (2020) 38(15):1000. doi: 10.1200/ JCO.2020.38.15_suppl.1000

41. Ko EC, Formenti SC. Radiation Therapy to Enhance Tumor Immunotherapy: A Novel Application for an Established Modality. Int J Radiat Biol (2019) 95 (7):936-9. doi: 10.1080/09553002.2019.1623429

42. Tabarestani S, Esmaeil-Akbari M, Namaki S. Novel Approaches to Immunotherapy in Triple Negative Breast Cancer. Int J Cancer Manage (2018) 11(12):e87024. doi: 10.5812/ijcm.87024

43. Zhu XD, Zhang QZ, Wang D, Liu CG, Han B, Yang JM. Expression of PD-L1 Attenuates the Positive Impacts of High-Level Tumor-Infiltrating Lymphocytes on Prognosis of Triple-Negative Breast Cancer. Cancer Biol Ther (2019) 20(8):1105-12. doi: 10.1080/15384047.2019.1595282

44. Vihervuori H, Autere TA, Repo H, Kurki S, Kallio L, Lintunen MM, et al. Tumor-Infiltrating Lymphocytes and CD8(+) T Cells Predict Survival of Triple-Negative Breast Cancer. J Cancer Res Clin Oncol (2019) 145(12):310514. doi: 10.1007/s00432-019-03036-5

45. Jin YW, Hu PZ. Tumor-Infiltrating CD8 T Cells Predict Clinical Breast Cancer Outcomes in Young Women. Cancers (2020) 12(5):1076. doi: 10.3390/ cancers 12051076

46. Guo L, Cao CM, Goswami S, Huang XY, Ma LXX, Guo YC, et al. Tumoral PD-1(Hi)CD8(+) T Cells Are Partially Exhausted and Predict Favorable Outcome in Triple-Negative Breast Cancer. Clin Sci (2020) 134(7):711-26. doi: $10.1042 / C s 20191261$ 
47. He Z, Khatib AM, Creemers JWM. Loss of the Proprotein Convertase Furin in $\mathrm{T}$ Cells Represses Mammary Tumorigenesis in Oncogene-Driven Triple Negative Breast Cancer. Cancer Lett (2020) 484:40-9. doi: 10.1016/ j.canlet.2020.05.001

48. Piccirillo CA, Shevach EM. Cutting Edge: Control of CD8(+) T Cell Activation by $\mathrm{CD} 4(+) \mathrm{CD} 25(+)$ Immunoregulatory Cells. J Immunol (2001) 167(3):1137-40. doi: 10.4049/jimmunol.167.3.1137

49. Carpenter KJ, Valfort AC, Steinauer N, Chatterjee A, Abuirqeba S, Majidi S, et al. LXR-Inverse Agonism Stimulates Immune-Mediated Tumor Destruction by Enhancing CD8 T-Cell Activity in Triple Negative Breast Cancer. Sci Rep (2019) 9:19530. doi: 10.1038/s41598-019-56038-1

50. Watkins-Schulz R, Tiet P, Gallovic MD, Junkins RD, Batty C, Bachelder EM, et al. A Microparticle Platform for STING-Targeted Immunotherapy Enhances Natural Killer Cell- and CD8(+) T Cell-Mediated Anti-Tumor Immunity. Biomaterials (2019) 205:94-105. doi: 10.1016/j.biomaterials. 2019.03.011

51. Pantelidou C, Sonzogni O, Taveira MD, Mehta AK, Kothari A, Wang D, et al. PARP Inhibitor Efficacy Depends on CD8(+) T-Cell Recruitment via Intratumoral STING Pathway Activation in BRCA-Deficient Models of Triple-Negative Breast Cancer. Cancer Discovery (2019) 9(6):722-37. doi: 10.1158/2159-8290.Cd-18-1218

52. Li Y, Xiao F, Zhang A, Zhang D, Nie W, Xu T, et al. Oncolytic Adenovirus Targeting TGF-Beta Enhances Anti-Tumor Responses of MesothelinTargeted Chimeric Antigen Receptor T Cell Therapy Against Breast Cancer. Cell Immunol (2020) 348:104041. doi: 10.1016/j.cellimm.2020.104041

53. Li ZH, Qiu YR, Lu WQ, Jiang Y, Wang J. Immunotherapeutic Interventions of Triple Negative Breast Cancer. J Trans Med (2018) 16:147. doi: 10.1186/ s12967-018-1514-7

54. Xia L, Zheng ZZ, Liu JY, Chen YJ, Ding JC, Xia NS, et al. EGFR-Targeted CAR-T Cells Are Potent and Specific in Suppressing Triple-Negative Breast Cancer Both In Vitro and In Vivo. Clin Transl Immunol (2020) 9(5):e01135. doi: $10.1002 / \mathrm{cti} 2.1135$

55. Wei H, Wang Z, Kuang Y, Wu ZG, Zhao SS, Zhang ZL, et al. Intercellular Adhesion Molecule-1 as Target for CAR-T-Cell Therapy of Triple-Negative Breast Cancer. Front Immunol (2020) 11:573823. doi: 10.3389/ fimmu.2020.573823

56. Tchou J, Zhao YB, Levine BL, Zhang PJ, Davis MM, Melenhorst JJ, et al. Safety and Efficacy of Intratumoral Injections of Chimeric Antigen Receptor (CAR) T Cells in Metastatic Breast Cancer. Cancer Immunol Res (2017) 5(12):115261. doi: 10.1158/2326-6066.Cir-17-0189

57. Zhou R, Yazdanifar M, Das Roy L, Whilding LM, Gavrill A, Maher J, et al. CAR T Cells Targeting the Tumor MUC1 Glycoprotein Reduce TripleNegative Breast Cancer Growth. Front Immunol (2019) 10:1149. doi: 10.3389/fimmu.2019.01149

58. Stuber T, Monjezi R, Wallstabe L. Inhibition of TGF-Beta-Receptor Signaling Augments the Antitumor Function of ROR1-Specific CAR T-Cells Against Triple-Negative Breast Cancer (Vol 8, E000676, 2020). J Immunother Cancer (2020) 8(1):e000676corr1. doi: 10.1136/jitc-2020-000676corr1

59. Dees S, Ganesan R, Singh S, Grewal IS. Emerging CAR-T Cell Therapy for the Treatment of Triple-Negative Breast Cancer. Mol Cancer Ther (2020) 19 (12):2409-21. doi: 10.1158/1535-7163.MCT-20-0385

60. Han YL, Xie W, Song DG, Powell DJ. Control of Triple-Negative Breast Cancer Using Ex Vivo Self-Enriched, Costimulated NKG2D CAR T Cells. J Hematol Oncol (2018) 11:92. doi: 10.1186/s13045-018-0635-Z

61. Hu ZW. Tissue Factor as a New Target for CAR-NK Cell Immunotherapy of Triple-Negative Breast Cancer. Sci Rep (2020) 10(1):2815. doi: 10.1038/ s41598-020-59736-3

62. Liu Y, Zhou YH, Huang KH, Fang XJ, Li Y, Wang FF, et al. Targeting Epidermal Growth Factor-Overexpressing Triple-Negative Breast Cancer by Natural Killer Cells Expressing a Specific Chimeric Antigen Receptor. Cell Prolif (2020) 53(8):2815. doi: 10.1111/cpr.12858

63. Cao W, Li JQ, Hao QY, Vadgama JV, Wu Y. AMP-Activated Protein Kinase: A Potential Therapeutic Target for Triple-Negative Breast Cancer. Breast Cancer Res (2019) 21:29. doi: 10.1186/s13058-019-1107-2

64. Gupta GK, Collier AL, Lee D, Hoefer RA, Zheleva V, van Reesema LSL, et al. Perspectives on Triple-Negative Breast Cancer: Current Treatment Strategies, Unmet Needs, and Potential Targets for Future Therapies. Cancers (2020) 12 (9):2392. doi: 10.3390/cancers 12092392
65. Thankamony AP, Murali R, Karthikeyan N, Varghese BA, Teo WS, McFarland A, et al. Targeting the Id1-Kif11 Axis in Triple-Negative Breast Cancer Using Combination Therapy. Biomolecules (2020) 10(9):1295. doi: 10.3390/biom 10091295

66. Meric-Bernstam F, Larkin J, Tabernero J, Bonini C. Enhancing Anti-Tumour Efficacy With Immunotherapy Combinations. Lancet (2021) 397 (10278):1010-22. doi: 10.1016/S0140-6736(20)32598-8

67. Rotte A, Jin JY, Lemaire V. Mechanistic Overview of Immune Checkpoints to Support the Rational Design of Their Combinations in Cancer Immunotherapy. Ann Oncol (2018) 29(1):71-83. doi: 10.1093/annonc/ mdx686

68. Mahoney KM, Rennert PD, Freeman GJ. Combination Cancer Immunotherapy and New Immunomodulatory Targets. Nat Rev Drug Discovery (2015) 14(8):561-84. doi: 10.1038/nrd4591

69. Melero I, Berman DM, Aznar MA, Korman AJ, Gracia JLP, Haanen J. Evolving Synergistic Combinations of Targeted Immunotherapies to Combat Cancer. Nat Rev Cancer (2015) 15(8):457-72. doi: 10.1038/ $\operatorname{nrc3973}$

70. Cortes J, Cescon DW, Rugo HS, Im SA, Yusof MM, Gallardo C, et al. KEYNOTE-355: Final Results From a Randomized, Double-Blind Phase III Study of First-Line Pembrolizumab Plus Chemotherapy vs Placebo Plus Chemotherapy for Metastatic TNBC. Ann Oncol (2021) 32:S1289-90. doi: 10.1016/j.annonc.2021.08.2089

71. Bonaventura P, Shekarian T, Alcazer V, Valladeau-Guilemond J, ValsesiaWittmann S, Amigorena S, et al. Cold Tumors: A Therapeutic Challenge for Immunotherapy. Front Immunol (2019) 10:168. doi: 10.3389/fimmu.2019.00168

72. Gruosso T, Gigoux M, Manem VSK, Bertos N, Zuo D, Perlitch I, et al. Spatially Distinct Tumor Immune Microenvironments Stratify TripleNegative Breast Cancers. J Clin Invest (2019) 129(4):1785-800. doi: 10.1172/ JCI96313

73. Li X, Luo L, Jiang M, Zhu C, Shi Y, Zhang J, et al. Cocktail Strategy for 'Cold' Tumors Therapy via Active Recruitment of CD8+ T Cells and Enhancing Their Function. J Control Release (2021) 334:413-26. doi: 10.1016/ j.jconrel.2021.05.002

74. Barsoum FS, Awad AS, Hussein NH, Eissa RA, El Tayebi HM. MALAT-1: LncRNA Ruling miR-182/PIG-C/mesothelin Triad in Triple Negative Breast Cancer. Pathol Res Pract (2020) 216(12):153274. doi: 10.1016/j.prp. 2020.153274

75. Lv J, Li P. Mesothelin as a Biomarker for Targeted Therapy. biomark Res (2019) 7(1):18. doi: 10.1186/s40364-019-0169-8

76. Del Bano J, Flores-Flores R, Josselin E, Goubard A, Ganier L, Castellano R, et al. A Bispecific Antibody-Based Approach for Targeting Mesothelin in Triple Negative Breast Cancer. Front Immunol (2019) 10:1593. doi: 10.3389/ fimmu.2019.01593

77. Fujiyuki T, Amagai Y, Shoji K, Kuraishi T, Sugai A, Awano M, et al. Recombinant SLAMblind Measles Virus Is a Promising Candidate for Nectin-4-Positive Triple Negative Breast Cancer Therapy. Mol TherapyOncolyt (2020) 19:127-35. doi: 10.1016/j.omto.2020.09.007

78. Gholami S, Chen CH, Gao S, Lou E, Fujisawa S, Carson J, et al. Role of MAPK in Oncolytic Herpes Viral Therapy in Triple-Negative Breast Cancer. Cancer Gene Ther (2014) 21(7):283-9. doi: 10.1038/cgt.2014.28

79. Bramante S, Koski A, Liikanen I, Vassilev L, Oksanen M, Siurala M, et al. Oncolytic Virotherapy for Treatment of Breast Cancer, Including TripleNegative Breast Cancer. Oncoimmunology (2016) 5(2):e1078057. doi: 10.1080/ 2162402X.2015.1078057

80. Clifton GT, Litton JK, Arrington K, Ponniah S, Ibrahim NK, Gall V, et al. Results of a Phase Ib Trial of Combination Immunotherapy With a CD8+T Cell Eliciting Vaccine and Trastuzumab in Breast Cancer Patients. Ann Surg Oncol (2017) 24(8):2161-7. doi: 10.1245/s10434-017-5844-0

81. Niavarani SR, Lawson C, Boudaud M, Simard C, Tai LH. Oncolytic Vesicular Stomatitis Virus-Based Cellular Vaccine Improves Triple-Negative Breast Cancer Outcome by Enhancing Natural Killer and CD8(+) T-Cell Functionality. J Immunother Cancer (2020) 8(1):e000465. doi: 10.1136/jitc2019-000465

82. Ghouse SM, Nguyen HM, Bommareddy PK, Guz-Montgomery K, Saha D. Oncolytic Herpes Simplex Virus Encoding IL12 Controls Triple-Negative Breast Cancer Growth and Metastasis. Front Oncol (2020) 10:384. doi: $10.3389 /$ fonc. 2020.00384 
83. Li XF, Gruosso T, Zuo DM, Omeroglu A, Meterissian S, Guiot MC, et al. Infiltration of CD8(+) T Cells Into Tumor Cell Clusters in Triple-Negative Breast Cancer. Proc Natl Acad Sci USA (2019) 116(9):3678-87. doi: 10.1073/ pnas. 1817652116

84. Amedei A, Asadzadeh F, Papi F, Vannucchi MG, Ferrucci V, Bermejo IA, et al. A Structurally Simple Vaccine Candidate Reduces Progression and Dissemination of Triple-Negative Breast Cancer. Iscience (2020) 23 (6):101250. doi: 10.1016/j.isci.2020.101250

85. Osada T, Hartman ZC, Wei JP, Lei GJ, Hobeika AC, Gwin WR, et al. Polyfunctional Anti-Human Epidermal Growth Factor Receptor 3 (AntiHER3) Antibodies Induced by HER3 Vaccines Have Multiple Mechanisms of Antitumor Activity Against Therapy Resistant and Triple Negative Breast Cancers. Breast Cancer Res (2018) 20:90. doi: 10.1186/s13058-018-1023-x

86. Safavi A, Kefayat A, Mahdevar E, Ghahremani F, Nezafat N, Modarressi MH. Efficacy of Co-Immunization With the DNA and Peptide Vaccines Containing SYCP1 and ACRBP Epitopes in a Murine Triple-Negative Breast Cancer Model. Hum Vaccines Immunotherapeut (2020) 17(1):22-34. doi: 10.1080/ 21645515.2020.1763693
Conflict of Interest: The authors declare that the research was conducted in the absence of any commercial or financial relationships that could be construed as a potential conflict of interest.

Publisher's Note: All claims expressed in this article are solely those of the authors and do not necessarily represent those of their affiliated organizations, or those of the publisher, the editors and the reviewers. Any product that may be evaluated in this article, or claim that may be made by its manufacturer, is not guaranteed or endorsed by the publisher.

Copyright (C) 2022 Qiu, Zhang, Yan, Xiao, Ma, Lin, Wu, Li, Wang, Liu, Ma and Ma. This is an open-access article distributed under the terms of the Creative Commons Attribution License (CC BY). The use, distribution or reproduction in other forums is permitted, provided the original author(s) and the copyright owner(s) are credited and that the original publication in this journal is cited, in accordance with accepted academic practice. No use, distribution or reproduction is permitted which does not comply with these terms. 